\title{
GENERIC FINITENESS OF EQUILIBRIUM PAYOFFS FOR BIMATRIX GAMES
}

"In Honour of Martin Shubik"

Andreu Mas-Colell

Universitat Pompeu Fabra, and

Barcelona GSE

ABSTRACT

It is shown that in any affine space of payoff matrices the equilibrium payoffs of bimatrix games are generically finite.

KEY WORDS Bimatrix Games, Generic Finiteness 
PROLOGUE (2003)

The paper that follows is based on notes written in December of 1990. I'm indebted to A. McLennan and W. Zame for helpful comments of those. See also Govindan and McLennan [2001]. The paper was written up and presented at the Cowles Foundation in February 1994. The text below is the text of 1994. The topic belongs to a general area that has always been of interest to Martin Shubik and, thus, it is for me a pleasure and an honour to offer this contribution to him.

\section{Introduction}

It is well known that if the entries of the normal form of a bimatrix game (in fact, of games with any number of players) can be perturbed independently then generically there is a finite number of equilibria (see Van Damme [1983] and Fudenberg-Tirole [1991]).

Kreps-Wilson [1982] criticized this result as not being very helpful when the normal form is derived from an extensive form since then many strategies lead to the same final node. In fact, even if the payoffs of the final nodes can be perturbed independently the finiteness of the number of equilibria is not a generic property, yet Kreps-Wilson showed that the finiteness of the number of equilibrium payoffs is a generic property in this case. In many applications this suffices.

However, the Kreps-Wilson criticism to the normal form result can be reiterated: it is very strong to assume that final nodes utilities can be perturbed independently. Typically, many final nodes will correspond to the same final position of a game and the most 
natural assumption may be that the final utilities depend on the position and not on the particular history of play (i.e. the node).

In this note it is showed that for bimatrix games it is still true that the number of equilibrium payoffs is generically finite, in whatever way the payoffs of final nodes are tied together by (linear) constraints.

The interest of this result is limited (or enhanced?) by the fact that it does not extend to more than two players. A. McLennan [1990] has a clever example to that effect.

\section{The Result}

Proposition: Let $U$ be an arbitrary affine space of matrices. Then for a.e. payoff matrices $U_{1}, U_{2} \in U$ for two players the number of equilibrium payoffs of the corresponding bimatrix game is finite.

Remark: It is indispensable for this result that $U$ be the same for the two players (interpretation: if two final nodes correspond to two different positions then one can perturb the payoffs of both players). This is clear: take two payoffs matrices $U_{1}, U_{2}$ with a continuum of payoff equilibrium. Then $U_{1}=\left\{U_{1}\right\}$ and $U_{2}=\left\{U_{2}\right\}$ are affine spaces violating the desired property.

\section{Proof of the Proposition}

Without loss of generality it is enough to show that almost every pair of payoff matrices has at most a unique completely mixed equilibrium payoff. In general we should choose $U_{1}, U_{2}$ such that all of its submatrices belong to the generic set for the projection of $U$ on the corresponding coordinates. 
First two preliminary Lemmas.

Lemma 1: Let $A$ be a convex set of matrices. Then the function $A \rightarrow$ rankA is constant a.e. on $A$.

\section{Proof: This follows from the analyticity of the}

determinant function. Let $k$ be the maximal rank over the matrices in $A$. Suppose that we fix a $A \in A$ with maximal rank. Say that its first $k$ columns are linearly independent. By Fubini's theorem it is enough to show that for all $B \in A$ and a.e. $\alpha \in[0,1]$ we have that $\operatorname{rank}(\alpha A+(1-\alpha) B)=k$. Let $C_{\alpha}$ be the matrix formed by the first $k$ columns of $\alpha A+(1-\alpha) B$. Define $f(\alpha)=\operatorname{det}\left(C_{\alpha}^{T} C_{\alpha}\right)$. Note that $f(1) \neq 0$ and that $f$ is a polynomial on $\alpha$. Hence for $f(\alpha) \neq 0$ for a.e. $\alpha$ and so $\operatorname{rank}(\alpha A+(1-\alpha) B)=k$ for a.e. $\alpha$.

$$
\begin{aligned}
& \text { Lemma 2: Let } A \text { be a convex set of matrices. Then either } \\
& \text { (1) } A v=e(=(1, \ldots, 1)) \text { has a solution for a.e. } A \in A \text {, or } \\
& \text { (2) } A v=e \text { does not have a solution for a.e. } A \in A .
\end{aligned}
$$

Proof: Let $k$ be the rank that prevails a.e. over $A$ (Lemma 1). Consider the (isometric to $A$ ) convex set of matrices $A^{\prime}=\{[A, e]: A \in A\} . \quad$ Then either the a.e. rank of $A^{\prime}$ is $k$ and then (1) holds, or it is $k+1$ and then (2) holds.

We now prove the Proposition. 
Consider any pair of payoff matrices $U_{1}, U_{2}$ with the property that, first, they belong to the generic set of Lemma 2 and, second, $U_{1}^{T}, U_{2}^{T}$ belong to the generic set of Lemma 2 for $U^{T}=\left\{A^{T}: A \in U\right\}$. If possibility (2) of Lemma 2 holds for $U_{1}$ and $U_{2}^{T}$ then either there is no completely mixed solution of the unique equilibrium payoff is $(0,0)$.

Suppose that possibility (1) of Lemma 2 holds for $U_{1}$ (hence for $U_{2}$ ). Then there is $v_{2}$ such that $U_{2} v_{2}=e$. Take now any solution $\left(p_{1}, \alpha\right)$ to $U_{2}^{T} p_{1}=\alpha e, p_{1} \cdot e=1$. Then:

$$
1=e^{T} \cdot p_{1}=v_{2} \cdot U_{2}^{T} p_{1}=\alpha\left(v_{2} \cdot e\right)
$$

and so the only (completely mixed) equilibrium payoff for player 2 is

$$
\alpha=\frac{1}{\left(v_{2} \cdot e\right)}
$$

Since then $\alpha \neq 0$ this also implies that possibility (1) of Lemma 2 holds for $U_{2}^{T}$ (hence for $U_{1}^{T}$ ). We now repeat the argument. Choose $v_{1}$ such that $U_{1}^{T} v_{1}=e$. Consider any solution $\left(p_{2}, \beta\right)$ to $U_{1} p_{2}=\beta e, p_{2} \cdot e=1 . \quad$ Then:

$$
1=e \cdot p_{2}=v_{1} \cdot U_{1} p_{2}=\beta\left(v_{1} \cdot e\right)
$$

and so the only (completely mixed) equilibrium payoff for player 1 is

$$
\beta=\frac{1}{\left(v_{1} \cdot e\right)}
$$

Finally, the argument is completely symmetric (in fact just a matter of labelling) if possibility (1) of Lemma 2 holds for $U_{2}^{T}$.

\section{References}

Fudenberg, D. and J. Tirole [1991] Game Theory Cambridge: MIT Press. 
Kreps, D and R. Wilson [1982] Sequential equilibrium, Econometrica, $50: 863-94$

Govindan, S. and A. McLennan [2001]. On the Generic Finiteness of Equilibrium Outcome Distributions in Game Forms,Econometrica, 69, $2: 455-472$.

McLennan, A. [1990] Private Communication. van Damme, E. [1983] Refinements of the Nash equilibrium concept, Springer-Verlag . 